\title{
Association of D4-GDI expression with breast cancer progression
}

\author{
Leslie A. Rivera Rosado ${ }^{\mathrm{a}}$, Jaime Rodriguez-Canales ${ }^{\mathrm{b}}$ and Baolin Zhang ${ }^{\mathrm{a}, *}$ \\ ${ }^{a}$ Division of Therapeutic Proteins, Office of Biotechnology Products, Center for Drug Evaluation and Research, \\ Food and Drug Administration, Bethesda, MD, USA \\ ${ }^{\mathrm{b}}$ Laboratory of Pathology, National Cancer Institute, National Institutes of Health, Bethesda, MD, USA
}

\begin{abstract}
D4-GDI is a key regulator of Rho GTPases that have been implicated in several aspects of breast tumorigenesis. We have previously found that D4-GDI was selectively expressed in breast cancer cell lines over normal mammary epithelial cells [45]. In this study, we investigated the expression level of D4-GDI in breast tumor specimens $(n=165)$ by immunohistochemistry using a validated antibody that specifically recognizes the full-length D4-GDI protein. D4-GDI was predominantly expressed in the luminal cells of the duct in contrast to the myoepithelial cells of the outer layer. The percentage of D4-GDI positive samples were found to be higher in the early stages of breast cancers followed by a significant decrease in malignant tumors and metastatic lesions when compared to normal breast tissues $(p<0.01)$. Analysis of matched samples confirmed the lower expression of D4-GDI in malignant tumors than normal adjacent tissues, while there was no further decrease in metastatic lesions. These results suggest that D4-GDI may function as a biphasic regulator of breast cancer progression and metastasis.
\end{abstract}

Keywords: D4-GDI, breast cancer, progression, immunohistochemistry

\author{
Abbreviations \\ RhoGDI, Rho GDP dissociation inhibitor (RhoG- \\ DI alpha); \\ D4-GDI, Rho GDP dissociation inhibitor beta \\ (RhoGDI beta or RhoGDI-2); \\ Rac1, Ras-related C3 botulinum toxin sub- \\ strate 1; \\ TMA, tissue microarray; \\ WT, wild type.
}

\section{Introduction}

Breast cancer is a heterogeneous disease that exhibits distinct molecular profiles and clinical outcomes. In addition to tumor size and nodal status, several molecular markers are being clinically used for diagnosis and de-

* Correspondence author: Baolin Zhang, Ph.D., 29 Lincoln Dr., Bldg. 29A, Rm. 2A01, HFD-122, Bethesda, MD 20892, USA. Tel.: +1 301827 1784; Fax: +1 301480 3256; E-mail: Baolin.Zhang@ fda.hhs.gov. sign of the individualized treatment. These include the estrogen receptors (ER), progesterone receptors (PR), and human epidermal growth factor receptor 2 (Her-2). Approximately $60-70 \%$ of breast cancers express ER and/or PR and 20-30\% of breast cancers have amplified Her-2 gene [4], which provide a basis for treatment with targeted therapies such as tamoxifen and trastuzumab. However, 15-20\% breast tumors do not express ER/PR or HER-2 and are thus defined as triple-negative breast cancer. This subtype of breast cancer is clinically characterized as more aggressive and less responsive to the standard treatment and is associated with poor patient prognosis $[4,6]$. Although gene expression signatures have been proposed by several groups for classification of breast tumor subtypes, the results are not generally applicable due to their significant discrepancy $[22,23$, 29,36,41].

Recently Rho GDP-dissociation inhibitors (RhoGDIs) have come to light as potential regulators of tumorigenesis and cancer progression [42]. The human RhoGDI family includes RhoGDI, D4-GDI, and RhoGDI-3. These proteins are crucial regulators of Rho GTPases, including Rac1, Cdc42, and RhoA, 
through which they control a variety of signaling pathways that are essential for normal cell function. Like RhoGDI, D4-GDI is thought to form stable complexes with individual Rho proteins thereby controlling their subcellular localization and cellular activities. Aberrant signaling through Rho GTPases has been implicated in virtually all aspects of tumorigenesis - tumorigenic transformation, outgrowth, metastasis and angiogenesis $[1,15]$. It has therefore been suggested that by modulating the activity of Rho GTPases, RhoGDIs could participate in the cellular processes responsible for tumorigenesis [42].

D4-GDI, also known as RhoGDI-2 or LyGDI or RhoGDI- $\beta$, was originally identified in hematopoietic tissues such as T- and B- cells [19,31]. However, increasing evidence shows that D4-GDI is also expressed in cells of non-hematopoietic neoplasms, including bladder [11], ovarian [37], colon [28], lung [27], and gastric [5] carcinoma. Our laboratory was the first to show that D4-GDI is selectively expressed in invasive breast cancer cell lines over normal mammary epithelial cells [45]. We have also demonstrated that silencing of D4-GDI gene abolished tumor growth and experimental metastasis in a xenograft mouse model [44].

This study was aimed at studying D4-GDI protein expression in primary breast tumors. Compared with normal breast tissues, D4-GDI protein is upregulated in the early stages of cancer progression (hyperplasia and benign) followed by a decrease in malignant tumors and metastatic lymph nodes. The results suggest that D4-GDI may play dual roles in regulation of breast cancer progression.

\section{Materials and methods}

\subsection{Cell lines and reagents}

All human breast cancer cell lines used in this study were obtained from the American Type Culture Collection (Manassas, VA), with the exception of SUM1315MO2 from Asterand biorepository (Detroit, MI). MDA-MB-231 derivatives stably expressing siRNA against human D4-GDI (siD4-GDI), Rho-GDI (siRho-GDI) or firefly luciferase (siCtrl) transcripts were described previously [45]. The cell lines were periodically ( $\sim 3$ months) tested for the absence of mycoplasma contamination in our laboratory. Rabbit polyclonal antibody against D4-GDI (N 1-20) and monoclonal antibodies specific to human Rho-GDI were from BD Biosciences, anti-D4-GDI mouse monoclon- al (IgG2b) clone 97A1015 was from Affinity BioReagents, anti-actin (I-19) goat polyclonal from Santa Cruz. Recombinant human TNF-related apoptosis inducing ligand (rhTRAIL) was from R \& D System.

\subsection{Western blotting}

Cells were lysed in Pierce IP lysis buffer (Thermo Scientific) for $30 \mathrm{~min}$ at $4{ }^{\circ} \mathrm{C}$. Protein concentrations of each whole cell lysate were determined using the BCA protein assay (Pierce, Rockford, IL). Equal amounts of cell lysates were resolved by electrophoresis using 4-12\% NuPAGE Bis-Tris gels (Invitrogen) and transferred to PVDF membranes (Millipore) for immunoblot analysis with an appropriate dilution of antibodies (1:100 to 1:2000). When necessary, the membranes were stripped by Restore western blot stripping buffer (Pierce) and reprobed with appropriate antibodies. Immunocomplexes were visualized by chemiluminescence using ECL reagent (Santa Cruz).

\subsection{Tissue microarrays}

Breast cancer tissue microarrays (TMAs; Cat No. BR2082, BR1003 and BR953) were obtained from US Biomax, Inc. (Rockville, MD). All the tissue samples were preserved in $10 \%$ phosphate buffered formalin ( $\mathrm{pH} \mathrm{7.4),} \mathrm{embedded} \mathrm{in} \mathrm{paraffin,} \mathrm{processed} \mathrm{into}$ sections. Array sections were mounted on the positive charged super plus glass slide. The tissue microarray sections were cut at $4 \mu \mathrm{m}$ think. Individual cores are $1.5 \mathrm{~mm}$ in diameter, spaced $0.25 \mathrm{~mm}$. The pathology diagnosis of tissues in TMAs was provided by US Biomax, and confirmed by the microscopic evaluation of the histopathology of the cores by two independent board certified pathologists. The tissue samples are classified into the following clinical relevant groups: Normal or NAT, normal breast tissue or normal adjacent to tumor; Hyperplasia; Benign, fibroadenoma; In Situ, intraductal and lobular carcinoma in situ; Malignant, invasive ductal and lobular carcinomas; Metastatic, breast cancer metastases to the lymph nodes. After histological evaluation by our pathologists the tissue microarrays contained: BR2082- 29 cases of metastatic carcinoma, 84 invasive carcinoma (malignant), 16 intraductal carcinoma and 3 lobular carcinoma in situ (In Situ), 8 fibroadenoma (benign), 4 of hyperplasia, 21 adjacent normal tissue and normal tissue, single core per case. BR1003- 100 tissue cores, duplicated cores of 10 cases of each type (hyperplasia, dysplasia, ductal carcinoma and lobular carcinoma) and triplicated cores of 6 breast normal tissues. BR956- Paired matched samples, 7 pairs of normal/ malignant and 9 pairs of malignant/metastatic, duplicated cores per case. 


\subsection{Immunohistochemistry}

Tissue sections were deparaffinized in xylene, and rehydrated in gradients of alcohol and water. Endogenous peroxidase activity was blocked by incubating slides in $3 \%$ hydrogen peroxide at room temperature for 5 minutes. Antigen retrieval was performed in the antigen unmasking solution, H3300, for 30 minutes in microwave oven. To reduce non-specific staining, slides were washed in phosphate buffered saline with Tween20 , followed by incubation in $2.5 \%$ normal horse blocking serum for $30 \mathrm{~min}$. Blocked sections were incubated in primary anti-D4-GDI polyclonal antibody (1:100) for 1 hour at room temperature. After washing three times, slides were incubated for 30 minutes with ImmPRESS anti-rabbit Ig reagent (Vector Laboratories) followed by incubation with the peroxidase substrate DAB solution (DAKO Cytomation) until desired stain intensity develops. Slides were counterstained with Hematoxylin and mounted with permanent mounting medium.

Qualitative/semi-quantitative scoring was independently done by two board certified pathologists. Staining intensities in cancer cells were compared with inflammatory cells (particularly lymphocytes) that are known to express high levels of D4-GDI that was used as internal positive control. The cells of stroma, collagen and adipose tissues showed minimal or no staining and were used as internal negative controls. The staining intensities when compared to negative and positive controls on the same tissue core were defined as: 0 , negative or background level staining; 1, weak staining; 2 , moderate intensity staining and 3 , strong intensity staining. The average staining intensity was calculated from the semi-quantitative scores given by each of the two pathologists or an average of duplicated cores accordingly.

\subsection{Statistical analysis}

Statistical analyses were performed using ANOVA followed by Bonferroni's multiple comparison test or Student's t test.

\section{Results}

\subsection{Anti-D4-GDI antibody validation}

In order to identify an appropriate anti-D4-GDI antibody for immunohistochemistry staining, we tested the specificity and selectivity of two commercially available anti-D4-GDI antibodies by western blot analysis using cell lysates from MDA-MB-231 cells that are known to express high levels of D4-GDI [45]. To test specificity, MDA-MB-231 breast cancer cells were treated with $20 \mathrm{ng} / \mathrm{ml}$ rhTRAIL for 4 hours in order to induce caspase activation [46]. Caspase activation in turn leads to D4-GDI ( $\sim 28 \mathrm{kDa})$ cleavage to give rise to a $23 \mathrm{kDa}$ fragment $[7,16,39]$. Western blot analysis using whole cell lysates from TRAIL-treated and untreated cells revealed that the rabbit polyclonal antibody (N 1-20) recognized specifically full-length D4GDI while antibody clone 97A1015 only recognized the $23 \mathrm{kDa}$ D4-GDI cleavage fragment (Fig. 1A). To establish selectivity, cell lysates from stable MDA-MB231 cell lines $[43,45]$ that were deficient in either D4GDI or its closely related family member, Rho-GDI, were immunoblotted with antibodies against Rho-GDI and D4-GDI (N 1-20). As shown in Fig. 1B, antiD4-GDI (N 1-20) antibody detected a band of around $28 \mathrm{kDa}$ in parental and Rho-GDI knockdown but not in the D4-GDI knockdown cell lysates. Anti-D4-GDI antibody clone 97A1015 has been previously used for immunostaining of D4-GDI expression in breast tissues [12]. However, our results indicate this antibody (97A1015) only recognizes the $23 \mathrm{kDa}$ D4-GDI fragment and therefore the results from $\mathrm{Hu}$ et al. [12] do not reflect the expression of full-length D4-GDI protein. We confirm that anti-D4-GDI antibody N 1-20 selectively and specifically recognizes full-length D4-GDI protein, thus it was chosen to use in the subsequent experiments.

\subsection{D4-GDI expression in breast cancer cell lines}

We have previously shown that D4-GDI is expressed in a panel of 8 breast cancer cell lines [45]. In order to correlate D4-GDI expression with established clinically relevant parameters of individual cell lines, we tested D4-GDI expression by immunoblotting an additional 16 breast cancer cell lines. Surprisingly, the expression of D4-GDI in these cell lines proved to be highly heterogeneous (Fig. 1C). When combined with the previous data, eight out of 24 (33\%) cell lines showed high, 8/24 showed moderate to low and 8/24 showed negative D4-GDI expression levels. There appears no systemic correlation between D4-GDI expression and the cellular parameters such as the status of ER and Her2 transcripts, and p53 mutational profiles (Table 1). 
A

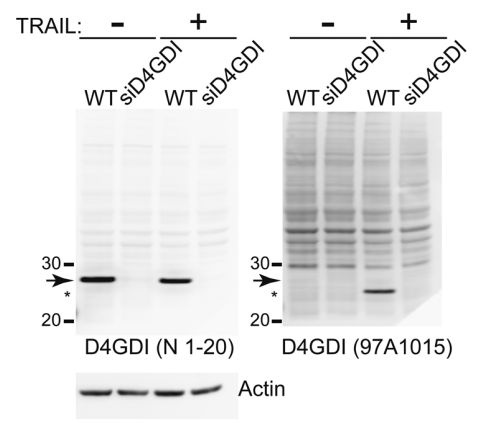

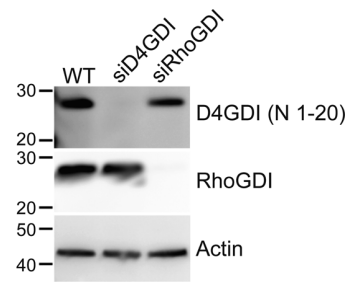

\section{C}
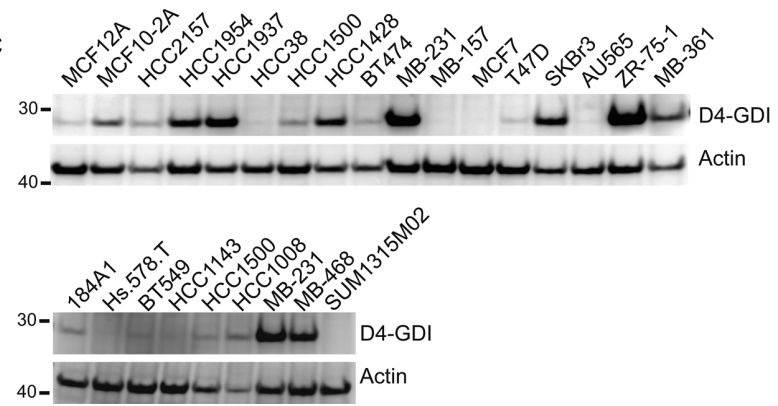

Fig. 1. Anti-D4-GDI antibody validation. A. Specificity of two distinct anti-D4-GDI antibodies. MDA-MB-231 cells, wild type or D4-GDI knockdown (siD4-GDI), were treated with 20ng/ml rhTRAIL for 2 hours. Cell lysates were immunoblotted using either anti-D4-GDI antibody (N 1-20) or anti-D4-GDI clone 97A1015. Equal loading was confirmed by reprobing with an anti-actin antibody. Arrow, full length D4-GDI; asterisk (*) D4-GDI cleavage fragment. B. Cell lysates from MDA-MB-231 (WT), D4-GDI knockdown (siD4GDI) or RhoGDI knockdown (siRhoGDI) cell lines were analyzed by immunoblotting to show selectivity of antibodies specific to either D4-GDI or RhoGDI. $C$. D4-GDI expression in human breast cancer cell lines. Equal amounts of whole cell proteins from each cell lines were immunoblotted with anti-D4-GDI N1-20 polyclonal antibody. Actin was used as an internal loading control.

\subsection{D4-GDI localization and expression during breast cancer progression}

Breast cancer progression is thought as a multistep process where normal epithelium transitions to nonatypical and atypical hyperplasia followed by carcinoma in situ and culminating in invasive carcinoma [2, $3,18]$. All the breast cancer cell lines used in detection of D4-GDI expression were derived from malignant tissues (see Table 1, tumor type), which do not reflect the stepwise disease stages. In order to address if D4-GDI expression is associated with the progression of breast cancer, we performed immunohistochemical staining for the full-length D4-GDI protein in human breast cancer tissue arrays containing 208 specimens (single core per case) from different disease stages, 165 of which were useful for analysis and scoring (43 specimens were either missing or non-evaluable). As a negative control, a duplicate array was stained with a normal rabbit serum. As illustrated in Fig. 2, D4-GDI staining was predominantly found in the luminal cells that line the breast ducts when compared with the my- oepithelial cells that surround the duct. Representative images from different tumor stages, staining intensities and their corresponding qualitative scores are shown in Fig. 3A. The immunostaining for D4-GDI in tumor cells was fairly homogenous in a specific tumor section. Therefore, we scored the D4-GDI staining for a tumor tissue as a whole. The average (mean \pm SD) D4-GDI staining intensities for the different stages of breast cancer progression were: normal or NAT $(n=21), 1.43 \pm$ 0.6 ; hyperplasia $(n=4), 2.13 \pm 0.25$; benign $(n=8)$, $1.56 \pm 0.73$; in situ $(n=19), 1.32 \pm 0.42$; malignant $(n=84), 0.79 \pm 0.7$ and metastasis $(n=29), 0.32 \pm$ 0.4 (Fig. 3B and Table 2). Overall, there was a slight increase on the average D4-GDI staining intensity in hyperplasia tissues when compared with normal tissues, and thereafter, significantly decreased in malignant tissue samples $(p<0.01)$ and metastatic lesions to the lymph nodes $(p<0.001)$. For an in-depth analysis, we calculated the percentage of samples in each stage that showed staining intensity higher than one $(>1)$, that is, the average intensity staining for normal breast tissue. As shown in Fig. 3C, the percentage (\%) of samples 
Table 1

Clinically relevant cellular markers of breast cancer cell lines

\begin{tabular}{|c|c|c|c|c|c|c|c|c|c|c|}
\hline Cell Line & D4-GDI ${ }^{\mathrm{a}}$ & $\begin{array}{c}\text { Gene } \\
\text { Cluster }^{\mathrm{b}}\end{array}$ & $\mathrm{ER}^{\mathrm{b}}$ & $\mathrm{PR}^{\mathrm{b}}$ & HER $2^{b}$ & TP53 $^{\mathrm{b}}$ & Source $^{b}$ & $\begin{array}{l}\text { Tumor } \\
\text { Type }^{\text {b }}\end{array}$ & $\begin{array}{c}\text { Metastatic } \\
\text { site }^{\mathrm{b}}\end{array}$ & $\begin{array}{l}\text { In vitro } \\
\text { Invasion }^{\mathrm{C}}\end{array}$ \\
\hline MCF12A & - & $\mathrm{BaB}$ & - & - & & + & P.Br & $\mathrm{F}$ & No & \\
\hline MCF10-2A & ++ & $\mathrm{BaB}$ & - & & & & $\mathrm{P} . \mathrm{Br}$ & $\mathrm{F}$ & No & \\
\hline HCC2157 & + & $\mathrm{BaA}$ & - & + & & + & P.Br & Duc.Ca & $1 / 9 \mathrm{LN}$ & \\
\hline HCC1954 & +++ & $\mathrm{BaA}$ & - & - & + & $+/-$ & P.Br & Duc.Ca & 0/27 LN & \\
\hline HCC1937 & +++ & $\mathrm{BaA}$ & - & - & & - & P.Br & Duc.Ca & NA & \\
\hline HCC38 & - & $\mathrm{BaB}$ & - & - & & $++\mathrm{M}$ & P.Br & Duc.Ca & $3 / 28 \mathrm{LN}$ & \\
\hline HCC1500 & + & $\mathrm{BaB}$ & $-/+$ & - & & - & P.Br & Duc.Ca & 4/24 LN & \\
\hline HCC1428 & +++ & $\mathrm{Lu}$ & + & + & & + & $\mathrm{PE}$ & Met. AC & $\mathrm{AC}$ and $\mathrm{PE}$ & \\
\hline BT474 & + & $\mathrm{Lu}$ & + & + & + & + & P.Br & IDC & & $\mathrm{L}$ \\
\hline MDA-MB-231 & ++++ & $\mathrm{BaB}$ & - & - & & $++\mathrm{M}$ & PE & $\mathrm{AC}$ & $\mathrm{PE}$ & $\mathrm{H}$ \\
\hline MDA-MB-157 & - & $\mathrm{BaB}$ & - & - & & - & PE & $\mathrm{MC}$ & PE & \\
\hline MCF7 & - & $\mathrm{Lu}$ & - & + & & $+/-\mathrm{WT}$ & $\mathrm{PE}$ & IDC & $\mathrm{PE}$ & $\mathrm{L}$ \\
\hline T47D & $-/+$ & $\mathrm{Lu}$ & + & + & & $++\mathrm{M}$ & $\mathrm{PE}$ & IDC & PE & $\mathrm{L}$ \\
\hline SKBR3 & +++ & $\mathrm{Lu}$ & - & - & + & + & PE & $\mathrm{AC}$ & $\mathrm{PE}$ & $\mathrm{L}$ \\
\hline AU565 & - & $\mathrm{Lu}$ & - & - & + & $+\mathrm{WT}$ & $\mathrm{PE}$ & $\mathrm{AC}$ & $\mathrm{PE}$ & \\
\hline ZR-75-1 & ++++ & $\mathrm{Lu}$ & + & - & & - & $\mathrm{AF}$ & IDC & $\mathrm{AF}$ & $\mathrm{L}$ \\
\hline MDA-MB-361 & ++ & $\mathrm{Lu}$ & + & - & + & $-\mathrm{WT}$ & P.Br & $\mathrm{AC}$ & Brain & $\mathrm{L}$ \\
\hline $184 \mathrm{~A} 1$ & - & $\mathrm{BaB}$ & - & & & & $\mathrm{RM}$ & & No & \\
\hline Hs.578.T & - & $\mathrm{BaB}$ & - & - & & & P.Br & $\mathrm{Ca}$ & No & $\mathrm{H}$ \\
\hline BT549 & + & $\mathrm{BaB}$ & - & - & & $++\mathrm{M}$ & P.Br & IDC. Pap & 3/7 LN & $\mathrm{H}$ \\
\hline HCC1143 & + & $\mathrm{BaA}$ & - & - & & +++ & $\mathrm{P} . \mathrm{Br}$ & Duc.Ca & $0 / 15 \mathrm{LN}$ & \\
\hline HCC1008 & +++ & $\mathrm{BaA}$ & - & - & + & +++ & $\mathrm{LN}$ & Met Duc.Ca & $12 / 15 \mathrm{LN}$ & \\
\hline MDA-MB-468 & +++ & $\mathrm{BaA}$ & - & - & & & $\mathrm{PE}$ & $\mathrm{AC}$ & PE & $\mathrm{L}$ \\
\hline SUM1315M02 & - & $\mathrm{BaB}$ & - & - & & + & Skin & IDC & Skin & \\
\hline
\end{tabular}

${ }^{\text {a }}$ 4-GDI expression level (from Fig. 1C) was determined by densitometry and normalized to actin expression.

${ }^{\mathrm{b}}$ Gene cluster, ER, PR, Her2 overexpression and TP53 expression as well as source, tumor type and metastasis information is from Neve RM et al. 2006 [26], Rahman M et al. 2008 [30], Kao J et al. 2009 [14], Kummerle NB et al. 2011 [17], Gazdar AF et al. 1998 [10] and www.atcc.org and references therein.

CIn vitro invasion data from Thompson EW et al. 1992 [40] and Sommers CL et al. 1994. [34].

Abbreviations: $\mathrm{AC}=$ Adenocarcinoma; $\mathrm{AF}=$ Ascites fluid; $\mathrm{BaA}=\mathrm{Basal} \mathrm{A}$ subtype; $\mathrm{BaB}=\mathrm{Basal} \mathrm{B}$ subtype; $\mathrm{Ca}=\mathrm{Carcinoma}$; Duc.Ca $=$ Ductal carcinoma; $\mathrm{F}=$ Fibrocystic dissease; $\mathrm{H}=$ High; IDC = Invasive ductal carcinoma; L = Low; LN = Lymph node; Lu = Luminal subtype; $\mathrm{M}=$ mutated $\mathrm{MC}=$ Medullary carcinoma; Met = Metastatic; NA = Not Available; Pap $=$ Papillary; $\mathrm{P} . \mathrm{Br}=\mathrm{Primary}$ breast tumor; $\mathrm{PE}=$ Pleural Effusion; RM = Reduction mammoplasty; WT = Wild type

that were positive $(>1)$ for D4-GDI staining increased during the hyperplasia stage, followed by a decrease in subsequent stages when compared to normal epithelium with a striking decrease in malignant tumors and falling to negative in metastatic lesions to the lymph node. Taken as a whole, the analysis of staining intensities by stage of disease progression reveals a biphasic expression pattern of D4-GDI. Consistent with the cell line data (Fig. 1C), D4-GDI staining intensity in malignant tumors was also heterogeneous (Fig. 3A). We then examined D4-GDI expression in Grade 1 and Grade 2 malignant tumors. The result shows that Grade 1 cohort expressed a slightly higher D4-GDI than Grade 2 cohort (Fig. 3D). However, a larger sample size is needed to address the heterogeneity of D4-GDI expression in malignant tumors. Since there was a drastic decrease in D4-GDI intensity in malignant and metastatic samples when compared to normal and adjacent tissue samples, we envision D4-GDI as a potential marker for breast cancer progression and that in combination with other established cellular markers could prove useful as a prognostic tool.

\subsection{D4-GDI expression during early pre-cancerous stages}

In order to confirm the upregulation of D4-GDI in the early stages of breast cancer progression we employed a tissue microarray that contained 100 cases of pre-cancerous disease and cancer with clinicopathological data. The data was grouped by disease stage and graphed against D4-GDI staining intensity (Fig. 4A and Table 3). This data confirms the results from an independent array (Fig. 3) that there is an increase in the percentage of D4-GDI positive samples in the hyperplasia and dysplasia (atypical hyperplasia) stages (Fig. 4B). Together these data suggest a positive role for D4-GDI early during breast tumorigenesis. 
Table 2

Average D4-GDI protein expression according to disease stage

\begin{tabular}{ccccccc}
\hline Average D4-GDI intensity & $\begin{array}{c}\text { Normal or NAT } \\
(n=21)\end{array}$ & $\begin{array}{c}\text { Hyperplasia } \\
(n=4)\end{array}$ & $\begin{array}{c}\text { Benign } \\
(n=8)\end{array}$ & $\begin{array}{c}\text { In situ } \\
(n=19)\end{array}$ & $\begin{array}{c}\text { Malignant } \\
(n=84)\end{array}$ & Metastasis \\
\hline 0 & 0 & 0 & 0 & 0 & $28(33 \%)$ & $18(62 \%)$ \\
0.5 & $2(9.5 \%)$ & 0 & $2(25 \%)$ & 0 & $7(8 \%)$ & $3(10 \%)$ \\
1 & $7(33 \%)$ & 0 & 0 & $10(53 \%)$ & $31(37 \%)$ & $8(28 \%)$ \\
1.5 & $6(28.5 \%)$ & 0 & $2(25 \%)$ & $7(37 \%)$ & $11(13 \%)$ & 0 \\
2 & $5(24 \%)$ & $3(75 \%)$ & $3(37.5 \%)$ & $1(5 \%)$ & $3(4 \%)$ & 0 \\
2.5 & 0 & $1(25 \%)$ & $1(12.5 \%)$ & $1(5 \%)$ & $4(5 \%)$ & 0 \\
3 & $1(5 \%)$ & 0 & 0 & 0 & 0 & 0 \\
\hline
\end{tabular}

The staining intensities when compared to negative and positive controls on the same tissue core were defined as: 0 , negative or background level staining; 1 , weak staining; 2 , medium intensity staining and 3, strong intensity staining. The average D4-GDI intensity was calculated by adding the scored from both pathologists and dividing it by two (Pathologist \#1 score + Pathologist \#2 score/2).

Normal or NAT, normal breast tissue or normal adjacent to tumor; Hyperplasia; Benign, fibroadenoma; In Situ, intraductal and lobular carcinoma in situ; Malignant, invasive ductal and lobular carcinomas; Metastatic, breast cancer metastases to the lymph nodes.

Table 3

Average D4-GDI protein expression in pre-cancerous and cancer stages

\begin{tabular}{ccccc}
\hline D4-GDI intensity & $\begin{array}{c}\text { Normal } \\
(n=5)\end{array}$ & $\begin{array}{c}\text { Hyperplasia } \\
(n=10)\end{array}$ & $\begin{array}{c}\text { Dysplasia } \\
(n=10)\end{array}$ & $\begin{array}{c}\text { Malignant } \\
(n=20)\end{array}$ \\
\hline 0 & $1(20 \%)$ & 0 & 0 & $1(5 \%)$ \\
0.5 & 0 & 0 & $1(10 \%)$ & $1(5 \%)$ \\
1 & $3(60 \%)$ & $5(50 \%)$ & $4(40 \%)$ & $16(80 \%)$ \\
1.5 & 0 & $3(30 \%)$ & $2(20 \%)$ & $1(5 \%)$ \\
2 & $1(20 \%)$ & $2(20 \%)$ & $1(10 \%)$ & $1(5 \%)$ \\
2.5 & 0 & 0 & $2(20 \%)$ & 0 \\
3 & 0 & 0 & 0 & 0 \\
\hline
\end{tabular}

The average D4-GDI intensity was calculated based on the staining intensities of duplicated cores per sample. The staining intensities were defined as: 0 , negative or background level staining; 1 , weak staining; 2 , medium intensity staining and 3 , strong intensity staining.

\subsection{D4-GDI expression in paired tissue samples}

Next we sought to examine if D4-GDI expression is lost during cancer progression using pair-matched samples (duplicated cores per case) from the same patients. Figure 5A shows representative images from normal and malignant pair-matched samples. The average D4-GDI staining intensity (mean \pm SD) for the normal samples was $1.7 \pm 0.15$ which was significantly higher than malignant samples $(0.9 \pm 0.32 ; p=0.04)$. There was an overall decrease in D4-GDI expression in malignant samples when compared to their corresponding pair-matched normal adjacent tissue. On the other hand, there was no significant difference between the expression of D4-GDI in malignant $(0.72 \pm 0.19$; mean $\pm \mathrm{SD})$ and metastatic $(0.77 \pm 0.15 ;$ mean $\pm \mathrm{SD})(p>$ $0.5)$ pair-matched samples (Fig. 5B). The percentages of D4-GDI positive samples in each group are graphed in Fig. 5C. This result shows a progressive decrease of D4-GDI protein expression through breast cancer

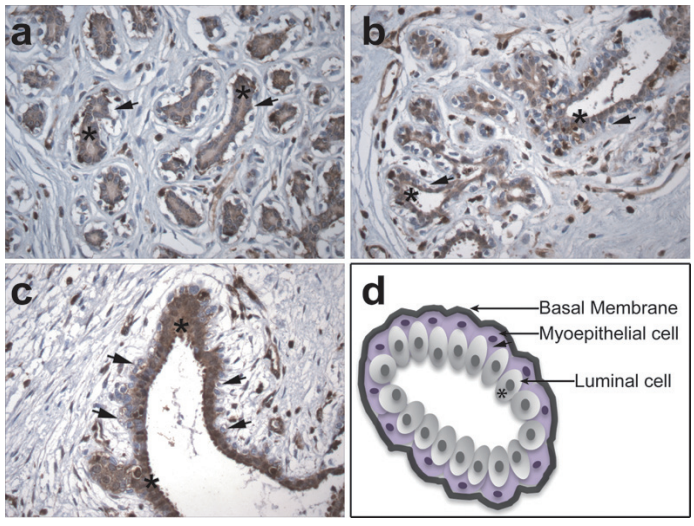

Fig. 2. Localization of D4-GDI expression. Immunohistochemical staining of human breast cancer tissue with anti-D4-GDI (N 1-20) rabbit polyclonal antibody (brown) and counterstained with Hematoxylin QS (blue). Immunoreactivity to D4-GDI was localized predominantly in the luminal cell population (asterisk, *) while the myoepithelial cell layer (arrows) was negative for D4-GDI staining. a, normal breast glandular epithelium. b, normal adjacent to tumor (NAT). c, benign tumor. d, illustration of a mammary duct.

progression into malignancy with no further significant decease during metastasis.

Given that there was a loss of D4-GDI expression in breast cancer metastases to the lymph nodes (Figs 3 and 5), we next examined a possible association between D4-GDI expression and lymph node positivity from the clinical data available. The mean D4-GDI staining intensity (mean $\pm \mathrm{SD}$ ), in samples that were known to have no lymph node involvement ( $n=11$ ) was 1.1 \pm 0.25 , while in lymph node positive samples ( $n=$ 18) was $0.83 \pm 0.15(p>0.5)$. There is no apparent correlation between loss of D4-GDI expression and lymph node involvement in this data set. 

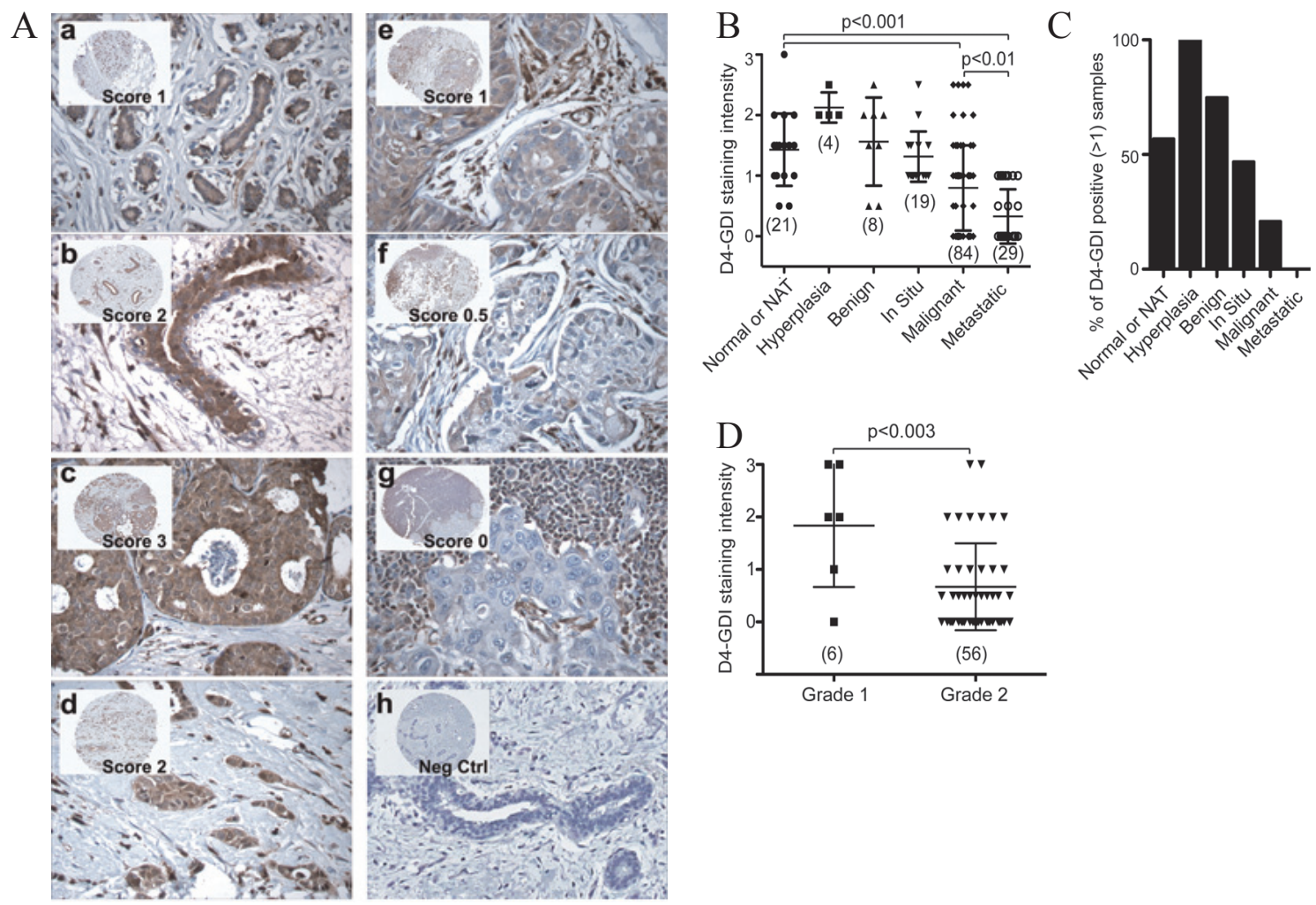

Fig. 3. D4-GDI protein expression in human breast cancer tissues. A. Immunohistochemical staining of D4-GDI in breast cancer progression tissue array (BR2082, US Biomax). Representative images from: a. normal, b. benign, c-f. malignant, g. lymph node metastasis and h. normal rabbit serum control, with their corresponding qualitative intensity scoring. (Normal or NAT, normal breast tissue or normal adjacent to tumor; Hyperplasia; Benign, fibroadenoma; In Situ, intraductal and lobular carcinoma in situ; Malignant, invasive ductal and lobular carcinomas; Metastatic, breast cancer metastases to the lymph nodes). B. Overall D4-GDI staining intensity distribution as a function on breast cancer progression. Each tissue sample was analyzed by two certified pathologist and given a semi-quantitative score based on staining intensity (y-axis), 0 - no staining, 1- weak staining, 2- medium staining and 3- strong staining and grouped by disease stage (x-axis). The average combined staining intensity scores are plotted (mean $\pm \mathrm{SD}$ ). $C$. The percentages $(\%)$ of samples that had D4-GDI intensity score higher than one $(>1)$ for each disease stage were plotted. D. Malignant samples with grade information (Grade 1 or Grade 2) were plotted against D4-GDI intensity staining (mean $\pm \mathrm{SD}$ ). Grade 1 or 2 is equivalent to well-differentiated, and moderately-differentiated, respectively.
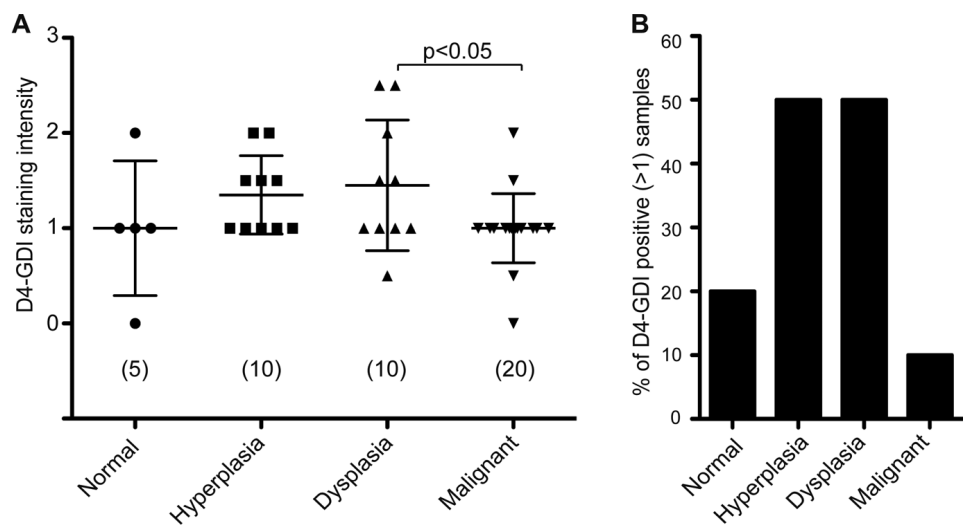

Fig. 4. D4-GDI expression during early stages of cancer progression. Human breast cancer tissue array (BR1003, US Biomax) containing duplicated cores of 10 cases of each type (hyperplasia, dysplasia [atypical hyperplasia], malignant [ductal carcinoma and lobular carcinoma] and triplicated cores of 7 breast normal tissues) was stained for D4-GDI expression. A. Samples were grouped by disease stage and plotted against D4-GDI staining intensity (mean \pm SD). $B$. The percentages $(\%)$ of D4-GDI positive $(>1)$ samples for each disease stage were plotted. 
A

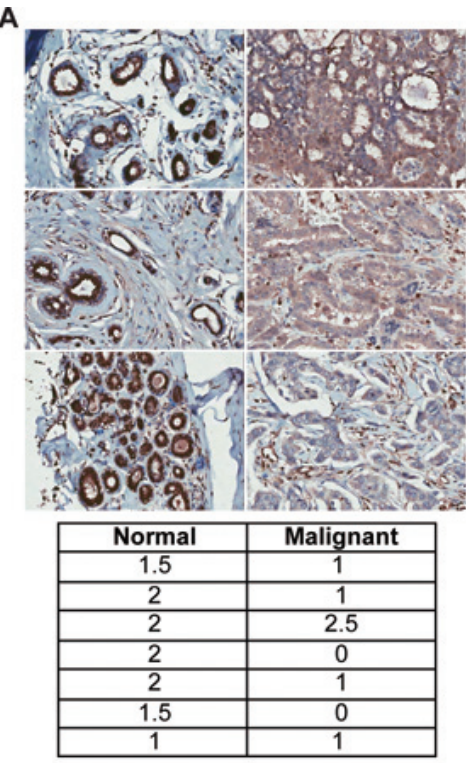

C

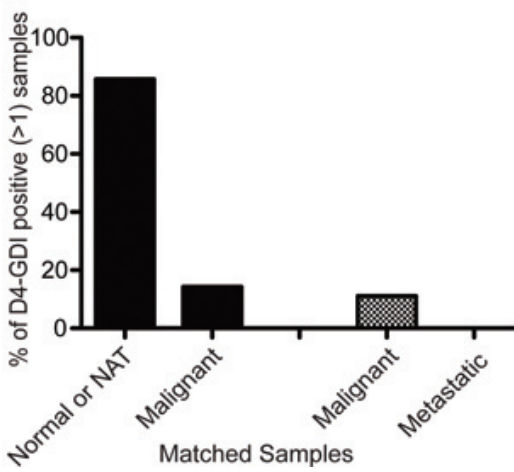

B

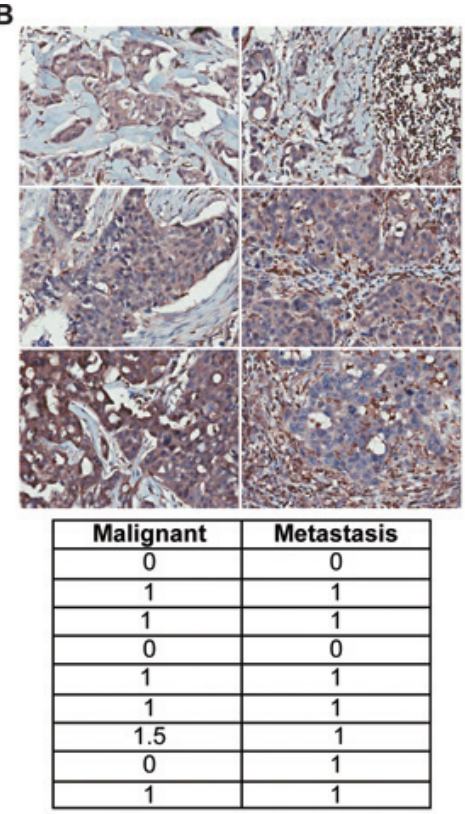

D

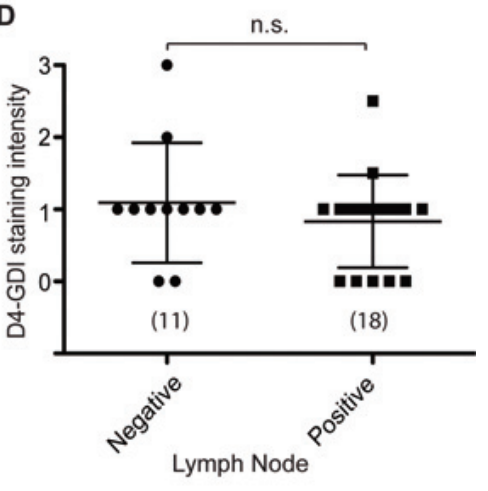

Fig. 5. D4-GDI expression in patient-matched tissue samples. Human breast cancer tissue array (BR953, US Biomax) containing duplicates of (A) normal and malignant $(n=7)$ and (B) malignant and metastatic $(n=8)$ matched samples was stained for D4-GDI. A. Normal tissue samples (left column) showed higher D4-GDI immunostaining than their malignant counterparts (right column). B. Malignant (left column) and metastatic (right column) tissue samples had low levels of D4-GDI staining. $C$. The percentages $(\%)$ of D4-GDI positive samples ( $>1)$ were plotted. D. Malignant tissue samples with available data for lymph node involvement were plotted against D4-GDI staining intensity (mean \pm $\mathrm{SD})$. There was non-statistical significance (n.s.) between the two groups.

\section{Discussion}

D4-GDI is a key regulator of Rho GTPases that have been implicated in several aspects of tumorigenesis, including malignant transformation and invasive phenotypes. In this study, we found an increased expression of D4-GDI in hyperplastic and benign stages of breast cancer when compared to normal mammary tissues, which was followed by a dramatic decrease in malignant tumors and metastatic lymph nodes.

D4-GDI was originally found in hematopoietic tissues such as T and B cells [19,31]. However, increasing evidence shows that D4-GDI is also expressed in nonhematopoietic neoplasms [5,9,11,20,27,37,38]. The elevated D4-GDI expression has been associated with tumor growth and malignant progression of ovarian [37], lung [27], gastric [5] and colorectal cancers [20]. In line with these observations, we have previously shown that D4-GDI is highly expressed in a panel of 8 breast cancer cell lines but not in normal mammary epithelial cells [45]. When a large number of cell lines were tested, however, the expression of D4-GDI appeared to be highly heterogeneous with no apparent correlation with any of the clinically relevant parameters (Table 1). 
This result promoted us to determine the expression of D4-GDI in primary breast tumors. The commercially available breast cancer progression tissue microarray used in our study contains samples that represent the stages of the current model for breast cancer progression. The multistep model of breast carcinogenesis suggests a transition from normal epithelia to non-atypical and atypical hyperplasia, evolving to carcinoma in situ and culminating in invasive carcinoma $[2,3,18]$. We found an increase in the percentage of D4-GDI positive samples in the hyperplasia and dysplasia stages when compared to normal breast or tumor adjacent tissues. Breast cancer is believed to develop mostly from cells of luminal lineage [21]. Notably, D4-GDI is predominantly expressed in the luminal cells of the mammary ducts but not in the myoepithelial cells at the outer layer. This observation, together with the increased D4-GDI expression during early stages of the disease, suggests that D4-GDI may promote tumorigenic transformation of mammary epithelial cells. In support of this notion, depletion of D4-GDI in MDA-MB-231 breast cancer cells suppressed invasive phenotypes both in vitro and in animal models $[44,45]$. Likewise, overexpression of D4-GDI in colorectal cancer cells promoted cell proliferation and in vitro motility and invasion [20]. On the other hand, our results show that the majority $(78 \%)$ of malignant breast cancer and all metastatic lymph nodes samples had little or undetectable D4-GDI immunoreactivity which is in agreement with reports by others [12,25,33]. Within the malignant samples, those that were grade 2 (moderately differentiated, data provided by US Biomax) tumors had the lowest D4-GDI expression (Fig. 3D). Given this observation, it will be of interest to study the expression of D4-GDI along the extent of cancer dedifferentiation and the possibility of D4-GDI playing a role in that process. The loss of D4GDI expression in malignant samples was confirmed by pair-sample analysis. Loss of D4-GDI expression in malignant breast cancers suggests a role for D4-GDI in preventing the development of a poorly-differentiated phenotype and/or tumor metastasis. In this regard, D4GDI has been proposed as a metastasis suppressor in bladder cancer [11,38]. Although our results reveal a similar pattern of D4-GDI expression as reported by $\mathrm{Hu}$ et al., we believe that the two groups are looking at two different forms of D4-GDI. While Hu et al. used an antibody that is now proved to recognize only the D4GDI cleavage fragment, we used the anti-D4-GDI N120 polyclonal that specifically detects the full-length D4-GDI protein in breast cancer cells.

The prognostic significance of D4-GDI expression in breast cancer is still controversial. cDNA microar- ray analysis showed no association between D4-GDI mRNA levels and clinical outcomes (e.g. disease-free or patient survival) $[13,33]$. However, careful consideration must be taken when analyzing D4-GDI mRNA levels as a measure of total amounts of D4-GDI protein in tumor tissues. Although it appears that D4-GDI protein expression correlates with the amount of D4-GDI RNA transcript in some cell lines [45], our unpublished results indicate that this is not always the case. Also, D4-GDI is highly expressed in hematopoietic cells, which may well be present in the heterogeneous tissue samples, which may cause contamination and even misleading results. We have directly measured D4-GDI protein expression in a large number of breast tissue and tumor samples ( $n=165$ ) using a validated, highly specific antibody to the full-length D4-GDI protein. Our results warrant further studies to determine how D4-GDI gene and protein expression is turned on in the luminal cells of mammary ducts and how it contributes to the malignant transformation.

D4-GDI appears to exhibit a biphasic pattern of expression along with breast cancer progression (Fig. 3). These seemingly contradictory results suggest that D4GDI may play dual roles in regulation of breast cancer cell functions depending on the genetic background associated with disease stages such as the status of Rho GTPases and their other regulatory proteins. In this regard, it has been shown that breast tumors have higher expression level of RhoA, Rac1 and Cdc42 compared to normal breast tissue [8,32]. As shown previously, D4-GDI binds preferentially to Rac GTPases in breast cancer cell lines [44]. By forming a stable complex, D4-GDI could either act as a Rac1 inhibitor by keeping the GTPase in its cytosolic, GDP-bound form [42] or as a Rac1 activator by shuttling the protein to membrane compartments where interacts and activate downstream targets [24,28]. Therefore, the dual roles of D4-GDI in breast cancer progression could be, at least in part, attributed to its dual role in Rac1 regulation. In addition, D4-GDI is known to be a substrate of caspase-3 and become cleaved in response to some physiological conditions such as fasting or stressful stimuli. The resulting fragment of D4-GDI (23 kDa) was detected in the breast tumor tissues. However, it is not clear whether cleavage of D4-GDI promotes or inhibits the activity of D4GDI protein. Studies are underway in our laboratory to investigate the functional outcome of D4-GDI cleavage, including its subcellular localization and binding affinity to Rac1 GTPase. Nonetheless, what it is clear from this study is that D4-GDI is over-expressed early during breast cancer progression and downregulated in 
malignant and lymph node metastatic lesions, suggesting a positive role for D4-GDI in tumorigenesis and a negative role during malignant transformation and/or metastasis.

In summary, D4-GDI protein is preferentially expressed in the luminal cells of the breast duct and at higher levels in hyperplasic and benign breast tumors. The expression of D4-GDI displays a biphasic pattern along with breast cancer progression i.e. it is increased in hyperplasia and benign tissues that is followed by a decrease in higher grades malignant cancers and metastatic lymph nodes. Although the prognostic significance of D4-GDI expression in breast cancer is still under debate, this study adds to the mounting amount of evidence suggesting a role for D4-GDI in breast cancer progression and metastasis.

\section{Author's contributions}

LRR performed Western blotting and statistical analysis, interpreted data, and assisted with manuscript preparation. JRC quantified immunostaining in breast tissue arrays and assisted with data analysis. BZ directed the design and implementation of the study and interpreted data. All authors read and approved the final manuscript.

\section{Conflicts of interests}

The authors declare that they have no conflicts of interests.

\section{Acknowledgements}

We thank Dr. John Gillespie from Pathology Consultant Services for tissue microarray analysis and scoring and helpful discussions on the pathology of breast tissues. This research was supported by the FDA Critical Path funding.

\section{References}

[1] S. Aznar, P. Fernandez-Valeron, C. Espina and J.C. Lacal, Rho GTPases: potential candidates for anticancer therapy, Cancer Lett 206 (2004), 181-191.

[2] M.W. Beckmann, D. Niederacher, H.G. Schnurch, B.A. Gusterson and H.G. Bender, Multistep carcinogenesis of breast cancer and tumour heterogeneity, J Mol Med (Berl) 75 (1997), 429-439.
[3] A. Bombonati and D.C. Sgroi, The molecular pathology of breast cancer progression, J Pathol 223 (2011), 307-317.

[4] J.D. Brenton, L.A. Carey, A.A. Ahmed and C. Caldas, Molecular classification and molecular forecasting of breast cancer: ready for clinical application? J Clin Oncol 23 (2005), 7350-7360.

[5] H.J. Cho, K.E. Baek, S.M. Park, I.K. Kim, Y.L. Choi, H.J. Cho, I.K. Nam, E.M. Hwang, J.Y. Park, J.Y. Han, S.S. Kang, D.C. Kim, W.S. Lee, M.N. Lee, G.T. Oh, J.W. Kim, C.W. Lee and J. Yoo, RhoGDI2 Expression Is Associated with Tumor Growth and Malignant Progression of Gastric Cancer, Clinical Cancer Research 15 (2009), 2612-2619.

[6] R. Dent, M. Trudeau, K.I. Pritchard, W.M. Hanna, H.K. Kahn, C.A. Sawka, L.A. Lickley, E. Rawlinson, P. Sun and S.A. Narod, Triple-negative breast cancer: clinical features and patterns of recurrence, Clin Cancer Res 13 (2007), 4429-4434.

[7] F. Essmann, T. Wieder, A. Otto, E.C. Müller, B. Dörken and P.T. Daniel, GDP dissociation inhibitor D4-GDI (Rho-GDI 2), but not the homologous rho-GDI 1 , is cleaved by caspase3 during drug-induced apoptosis, Biochemical Journal 346 (2000), 777-783.

[8] G. Fritz, I. Just and B. Kaina, Rho GTPases are over-expressed in human tumors, Int J Cancer 81 (1999), 682-687.

[9] A. Fujita, A. Shida, S. Fujioka, H. Kurihara, T. Okamoto and K. Yanaga, Clinical significance of Rho GDP dissociation inhibitor 2 in colorectal carcinoma, Int J Clin Oncol (2011).

[10] A.F. Gazdar, V. Kurvari, A. Virmani, L. Gollahon, M. Sakaguchi, M. Westerfield, D. Kodagoda, V. Stasny, H.T. Cunningham, Wistuba, II, G. Tomlinson, V. Tonk, R. Ashfaq, A.M. Leitch, J.D. Minna and J.W. Shay, Characterization of paired tumor and non-tumor cell lines established from patients with breast cancer, Int J Cancer 78 (1998), 766-774.

[11] J.J. Gildea, M.J. Seraj, G. Oxford, M.A. Harding, G.M. Hampton, C.A. Moskaluk, H.F. Frierson, M.R. Conaway and D. Theodorescu, RhoGDI2 is an invasion and metastasis suppressor gene in human cancer, Cancer Res 62 (2002), 6418-6423.

[12] L.D. Hu, H.F. Zou, S.X. Zhan and K.M. Cao, Biphasic expression of RhoGDI2 in the progression of breast cancer and its negative relation with lymph node metastasis, Oncol Rep 17 (2007), 1383-1389.

[13] W.G. Jiang, G. Watkins, J. Lane, G.H. Cunnick, A. DouglasJones, K. Mokbel and R.E. Mansel, Prognostic Value of Rho GTPases and Rho Guanine Nucleotide Dissociation Inhibitors in Human Breast Cancers, Clinical Cancer Research 9 (2003), 6432-6440.

[14] J. Kao, K. Salari, M. Bocanegra, Y.L. Choi, L. Girard, J. Gandhi, K.A. Kwei, T. Hernandez-Boussard, P. Wang, A.F. Gazdar, J.D. Minna and J.R. Pollack, Molecular profiling of breast cancer cell lines defines relevant tumor models and provides a resource for cancer gene discovery, PLoS One 4 (2009), e6146.

[15] R. Karlsson, E.D. Pedersen, Z. Wang and C. Brakebusch, Rho GTPase function in tumorigenesis, Biochim Biophys Acta 1796 (2009), 91-98.

[16] R.J. Krieser and A. Eastman, Cleavage and nuclear translocation of the caspase 3 substrate Rho GDP-dissociation inhibitor, D4-GDI, during apoptosis, Cell Death Differ 6 (1999), 412-419.

[17] N.B. Kuemmerle, E. Rysman, P.S. Lombardo, A.J. Flanagan, B.C. Lipe, W.A. Wells, J.R. Pettus, H.M. Froehlich, V.A. Memoli, P.M. Morganelli, J.V. Swinnen, L.A. Timmerman, L. Chaychi, C.J. Fricano, B.L. Eisenberg, W.B. Coleman and W.B. Kinlaw, Lipoprotein lipase links dietary fat to solid tumor cell proliferation, Mol Cancer Ther 10 (2011), 427-436. 
[18] S.R. Lakhani, The transition from hyperplasia to invasive carcinoma of the breast, J Pathol 187 (1999), 272-278.

[19] J.M. Lelias, C.N. Adra, G.M. Wulf, J.C. Guillemot, M. Khagad, D. Caput, and B. Lim, cDNA cloning of a human mRNA preferentially expressed in hematopoietic cells and with homology to a GDP-dissociation inhibitor for the rho GTPbinding proteins, Proc Natl Acad Sci U S A 90 (1993), 14791483.

[20] X. Li, J. Wang, X. Zhang, Y. Zeng, L. Liang and Y. Ding, Overexpression of RhoGDI2 Correlates with Tumor Progression and Poor Prognosis in Colorectal Carcinoma, Ann Surg Oncol (2011).

[21] E. Lim, F. Vaillant, D. Wu, N.C. Forrest, B. Pal, A.H. Hart, M.L. Asselin-Labat, D.E. Gyorki, T. Ward, A. Partanen, F. Feleppa, L.I. Huschtscha, H.J. Thorne, S.B. Fox, M. Yan, J.D. French, M.A. Brown, G.K. Smyth, J.E. Visvader and G.J. Lindeman, Aberrant luminal progenitors as the candidate target population for basal tumor development in BRCA1 mutation carriers, Nat Med 15 (2009), 907-913.

[22] F. Liu and B. Wu, Multi-group cancer outlier differential gene expression detection, Comput Biol Chem 31 (2007), 65-71.

[23] A.J. Minn, G.P. Gupta, P.M. Siegel, P.D. Bos, W. Shu, D.D. Giri, A. Viale, A.B. Olshen, W.L. Gerald and J. Massague, Genes that mediate breast cancer metastasis to lung, Nature 436 (2005), 518-524

[24] K. Moissoglu, K.S. McRoberts, J.A. Meier, D. Theodorescu and M.A. Schwartz, Rho GDP dissociation inhibitor 2 suppresses metastasis via unconventional regulation of RhoGTPases, Cancer Res 69 (2009), 2838-2844.

[25] H.G. Moon, S.H. Jeong, Y.T. Ju, C.Y. Jeong, J.S. Lee, Y.J. Lee, S.C. Hong, S.K. Choi, W.S. Ha, S.T. Park and E.J. Jung, Up-regulation of RhoGDI2 in human breast cancer and its prognostic implications, Cancer Res Treat 42 (2010), 151-156.

[26] R.M. Neve, K. Chin, J. Fridlyand, J. Yeh, F.L. Baehner, T. Fevr, L. Clark, N. Bayani, J.P. Coppe, F. Tong, T. Speed, P.T. Spellman, S. DeVries, A. Lapuk, N.J. Wang, W.L. Kuo, J.L. Stilwell, D. Pinkel, D.G. Albertson, F.M. Waldman, F. McCormick, R.B. Dickson, M.D. Johnson, M. Lippman, S. Ethier, A. Gazdar and J.W. Gray, A collection of breast cancer cell lines for the study of functionally distinct cancer subtypes, Cancer Cell 10 (2006), 515-527.

[27] H. Niu, H. Li, C. Xu and P. He, Expression profile of RhoGDI2 in lung cancers and role of RhoGDI2 in lung cancer metastasis, Oncol Rep 24 (2010), 465-471.

[28] T. Ota, M. Maeda, S. Suto and M. Tatsuka, LyGDI functions in cancer metastasis by anchoring Rho proteins to the cell membrane, Mol Carcinog 39 (2004), 206-220.

[29] C.M. Perou, T. Sorlie, M.B. Eisen, R.M. van de, S.S. Jeffrey, C.A. Rees, J.R. Pollack, D.T. Ross, H. Johnsen, L.A. Akslen, O. Fluge, A. Pergamenschikov, C. Williams, S.X. Zhu, P.E. Lonning, A.L. Borresen-Dale, P.O. Brown and D. Botstein, Molecular portraits of human breast tumours, Nature 406 (2000), 747-752.

[30] M. Rahman, S.R. Davis, J.G. Pumphrey, J. Bao, M.M. Nau, P.S. Meltzer, and S. Lipkowitz, TRAIL induces apoptosis in triple-negative breast cancer cells with a mesenchymal phenotype, Breast Cancer Res Treat 113 (2009), 217-230.

[31] P. Scherle, T. Behrens and L.M. Staudt, Ly-GDI, a GDPdissociation inhibitor of the RhoA GTP-binding protein, is expressed preferentially in lymphocytes, Proc Natl Acad Sci U S A 90 (1993), 7568-7572.

32] A. Schnelzer, D. Prechtel, U. Knaus, K. Dehne, M. Gerhard, H. Graeff, N. Harbeck, M. Schmitt and E. Lengyel, Rac1 in human breast cancer: overexpression, mutation analysis, and characterization of a new isoform, Rac1b, Oncogene 19 (2000), 3013-3020.

[33] D. Schunke, P. Span, H. Ronneburg, A. Dittmer, M. Vetter, H.J. Holzhausen, E. Kantelhardt, S. Krenkel, V. Muller, F.C. Sweep, C. Thomssen, and J. Dittmer, Cyclooxygenase-2 is a target gene of rho GDP dissociation inhibitor beta in breast cancer cells, Cancer Res 67 (2007), 10694-10702.

[34] C.L. Sommers, S.W. Byers, E.W. Thompson, J.A. Torri and E.P. Gelmann, Differentiation state and invasiveness of human breast cancer cell lines, Breast Cancer Res Treat 31 (1994), 325-335.

[35] D. Sun, D. Xu and B. Zhang, Rac signaling in tumorigenesis and as target for anticancer drug development, Drug Resistance Updates 9 (2006), 274-287.

[36] L.J. t Veer and D. De Jong, The microarray way to tailored cancer treatment, Nat Med 8 (2002), 13-14

[37] J. Tapper, E. Kettunen, W. El Rifai, M. Seppala, L.C. Andersson and S. Knuutila, Changes in gene expression during progression of ovarian carcinoma, Cancer Genet Cytogenet 128 (2001), 1-6.

[38] D. Theodorescu, L.M. Sapinoso, M.R. Conaway, G. Oxford, G.M. Hampton, and H.F. Frierson, Jr., Reduced expression of metastasis suppressor RhoGDI2 is associated with decreased survival for patients with bladder cancer, Clin Cancer Res $\mathbf{1 0}$ (2004), 3800-3806.

[39] B. Thiede, F. Siejak, C. Dimmler and T. Rudel, Prediction of translocation and cleavage of heterogeneous ribonuclear proteins and Rho guanine nucleotide dissociation inhibitor 2 during apoptosis by subcellular proteome analysis, Proteomics 2 (2002), 996-1006.

[40] E.W. Thompson, S. Paik, N. Brunner, C.L. Sommers, G. Zugmaier, R. Clarke, T.B. Shima, J. Torri, S. Donahue, M.E. Lippman et al., Association of increased basement membrane invasiveness with absence of estrogen receptor and expression of vimentin in human breast cancer cell lines, $J$ Cell Physiol 150 (1992), 534-44.

[41] M.A. Thorat, Gene-signature-based prognostic tools in breast cancer: not yet, Lancet 369 (2007), 1428.

[42] B. Zhang, Rho GDP dissociation inhibitors as potential targets for anticancer treatment, Drug Resist Updat 9 (2006), 134141.

[43] B. Zhang, Y. Zhang, M.C. Dagher and E. Shacter, Rho GDP dissociation inhibitor protects cancer cells against druginduced apoptosis, Cancer Res 65 (2005), 6054-6062.

[44] Y. Zhang, L.A. Rivera Rosado, S.Y. Moon and B. Zhang, Silencing of D4-GDI inhibits growth and invasive behavior in MDA-MB-231 cells by activation of Rac-dependent p38 and JNK signaling, J Biol Chem 284 (2009), 12956-12965.

[45] Y. Zhang and B. Zhang, D4-GDI, a Rho GTPase regulator, promotes breast cancer cell invasiveness, Cancer Res 66 (2006), 5592-5598.

[46] Y. Zhang and B. Zhang, TRAIL resistance of breast cancer cells is associated with constitutive endocytosis of death receptors 4 and 5, Mol Cancer Res 6 (2008), 1861-1871. 